\title{
ESTADO ACTUAL DE LA SALUD AFECTIVO-SEXUAL DE LAS PERSONAS CON DIVERSIDAD FUNCIONAL EN ESPAÑA
}

\author{
María Honrubia Pérez \\ Escuela de Enfermería de la Univesidad de Barcelona \\ mhonrubia@ub.edu \\ Marta Hernández Meroño \\ Vocal Asociación Nacional de Salud Sexual y Discapacidad (ANSSYD) \\ mhm130@gmail.com \\ Esther Sánchez Raja \\ Presidenta de la Asociación Nacional de Salud Sexual y Discapacidad (ANSSYD) \\ esanchezra@gmail.com
}

Fecha de Recepción: 4 Febrero 2018

Fecha de Admisión: 10 Abril 2018

\section{RESUMEN}

El objetivo de este estudio es aportar conocimiento sobre el estado actual de la Salud Afectivo Sexual de las personas con Diversidad Funcional (PDF) en el territorio español a partir de las consultas a la Asesoria de ANSSYD. Para ello se realiza un diseño cuantitativo descriptivo transversal, donde los sujetos de estudio sonPDF, familiares y profesionales que contactan con la asesoría. Los datos se obtienena partir de las consultas realizadas via online, telefónica durante 6 años. (Octubre de 2012 a Octubre de 2017).Para el registro de los datos se ha utilizado el paquete Microsofot Office 2010.

Resultados: Se atienden 1856 consultas de familiares, cuidadores y PDF, centrando sus dudas en las siguientes variables: Embarazo, desconocimiento sobre sexualidad, disfunciones sexuales, coito, orientación sexual, juguetes eróticos, asistencia sexual (AS), desconocimiento de las funciones del AS equiparándolo a terapia. En referencia a las consultas planteadas por profesionales se recogen 1758 consultas centrando sus dudas en las siguientes variables: anticoncepción, desconocimiento y dudas sobre sexualidad en PDF, Masturbación, coito, orientación sexual, juguetes eróticos, asistencia sexual (AS), fantasías y Derechos sexuales y Reproductivos de las PDF Conclusiones: Es patente el aumento de consultas recibidas en la Asesoría que se centran en intereses informativos sobre sexualidad que no han sido cubiertos por los profesionales a su servicio. La falta de desarrollo de la Ley Orgánica 2/2010 de 3 de Marzo de Salud Sexual y Reproductiva e interrupción voluntaria del embarazo tiene como consecuencia el incumplimiento de la misma con las consecuencias de falta de formación e información. La escasez de formación en sexualidad y más concretamente de sexualidad y diversidad funcional en los profesionales para afrontar las nece- 
sidades de las PDF y la carencia de programas y materiales educativos adaptados a las necesidades especiales de las PDF, dificulta el aprendizaje a este colectivo, a sus familias y a los profesionales en relación a la sexualidad, impidiendo a estas personas el empoderamiento a través de ella. Han de tomarse medidas urgentes por parte de las Administraciones, de los Ministerios de Educación y Sanidad y de los profesionales que ostentan cargos de gestión, para que en el siglo XXI los problemas de visualización y de reconocimiento de la afectividad y sexualidad en las PDF dejen de producirse.

Palabras clave: sexualidad; salud sexual; diversidad funcional; discapacidad; asesoría en sexualidad

\section{ABSTRACT}

Current state of the affective-sexual health of people with functional diversity in Spain.

The aim of this study is to contribute knowledge on the current Affective Sexual condition of the Health of the persons with Functional Diversity (PFD) in the Spanish territory through consultations to ANSSYD's Counseling. For that there is realized a quantitative descriptive transverse design, where the subjects of study are PFD, family and professionals that contact the advising. The data is obtained from the realized consultations online, telephonic for 6 years. (October 2012 to October 2017). For registration of the information has been used the package Microsoft Office 2010.

Results: There are attended 1856 consultations of family, keepers and PFD, centering his doubts on the following variables: Pregnancy, sexuality ignorance, sexual dysfunctions, coitus, sexual orientation, erotic toys, sexual assistance (SA), and ignorance of the functions of the SA comparing it to therapy. In reference to the consultations raised by professionals his doubts are 1758 focusing in the following variables: contraception, ignorance and doubts on sexuality in PFD, Masturbation, coitus, sexual orientation, erotic toys, sexual assistance SA, fantasies and sexual and Reproductive Laws of the PFD

Conclusions: Is evident the increase of consultations received in the Counseling that focuses on information interests on sexuality that have not been covered by the professionals at their service. The absence of development of the Organic Law 2/2010 of March 3 of Sexual and Reproductive Health and voluntary interruption of the pregnancy has the consequence noncompliance of it with consequences of lack of formation and information. The shortage of formation in sexuality and more concretely of sexuality and functional diversity in the professionals to confront the needs of the PFD and the lack of programs and educational materials adapted to the special needs of the PFD, makes learning difficult to this group, to his families and to the professionals in relation to the sexuality, preventing these persons the empowerment across her. Urgent measurements have to be taken by the Administrations, the Departments of Education and Health and the professionals who show charges of management, in order that in the 21 st century the problems of visualization and of recognition of the affectability and sexuality in the PDF stop taking place.

Keywords: sexuality; sexual health; functional diversity; disability: advising sexuality

\section{INTRODUCCIÓN}

"... La investigación en el ámbito de la discapacidad no debe considerarse como un conjunto de procedimientos técnicos y objetivos que realizan los expertos, sino como parte de la lucha de las personas con discapacidad por cuestionar la opresión que actualmente sufren en sus vidas cotidianas."

(Oliver, M. 2008). 
La Asociación Nacional de Salud Sexual y Discapacidad (ANSSYD)constituida y registrada en el Censo Nacional de Asociaciones, como entidad sin ánimo de lucro, abarca los entornos social, sanitario y educativo a los que tienen acceso las personas con discapacidad /Diversidad Funcional, dirigida por profesionales interesados en la promoción y defensa de sus derechos sexuales y reproductivos para el fomento de la Calidad de Vida. Sensible a estas necesidades y derechos, trabaja activamente entre otras cosas en colaborar para que se cumpla la legislación vigente y se dote a las personas en formación y a los profesionales de estrategias y herramientas que ayuden a mejorar la sexualidad de las personas con discapacidad.

En ANSSYD se trabaja la salud sexual con perspectiva interdisciplinar, desde cuatro ejes: Formación, Investigación, Asesoría en sexualidad y Divulgación informativa a la sociedad, además de tener presencia activa en las campañas sobre derechos, en la universalización de los métodos anticonceptivos, la educación sexual, la expresión de la sexualidad en la diversidad y la mejora de los servicios de atención a esta población.

Con la finalidad de favorecer la información y formaciónen en sexualidad de las personas con diversidad funcional (PDF) nace la Asesoría en Sexualidad para PDF, profesionales y familiares, que está atendida por profesionales de diversos ámbitos especializados en Sexualidad y Diversidad Funcional, así como en las alteraciones sexuales producidas por enfermedades crónicas

\section{TEMÁTICA PRINCIPAL}

El trabajo que se presenta es una investigación centrada en la actividad de uno de los Ejes en Ios que se fundamenta el trabajo de ANSSYD, la Asesoria en Sexualidad. La Asesoría gratuita online, telefónica y/o via skype de sexualidad y discapacidad a la que se dirigen tanto profesionales como familiares y las mismas personas con discapacidad a solicitar información y/o resolución de problemáticas referentes a su actividad sexual o de relación, es uno de los espacios creados que tiene la Asociación, como vía de comunicación con la sociedad. Desde el año 2012 se han venido registrando las consultas recibidas, estas han ido aumentando progresivamente y son contestadas por profesionales de la comunidad científica miembros de nuestra Asocición, con formación específica en sexualidad y discapacidad. Ha generado en los últimos seis años una gran demanda como consta en los datos que se presentan en el trabajo de investigación que se expone, datos que se consideran relevantes y constatan además de la importancia que la sexualidad tiene para estas personas y el entorno que las envuelve, la necesidad cada vez más urgente de visibilizar las necesidades sexuales de las personas con DF.

Los marcos teóricos de referencia en los que se fundamenta la actividad desarrollada por ANSSYD en referencia a las demandas de la Asesoría, son el Modelo Social descrito por Palacios(2008) y el Modelo Biográfico Profesional referenciado por Lameiras,Carrera, y Rodríguez, (2008), en el marco de la Convención de Derechos de las Personas con Discapacidad (2006), que celebró la Organización de Naciones Unidas, en New York en el año 2006 y ratificada por el Gobierno Español en el año 2008.

El Modelo Social sobre la discapacidad Victoria (2013), se enmarca en los principios generales declarados por los Derechos Humanos. Se origina en la segunda mitad del siglo pasado, dentro de la disciplina de las ciencias sociales, del análisis de las políticas sociales y la lucha por los derechos civiles relacionados con los derechos de las personas con discapacidad Oliver,(1998). Para este Modelo, las causas que originan la discapacidad no son religiosas ni científicas, sino sociales y las personas con discapacidad son suceptibles de aportar a la sociedad en igual medida que el resto de personas, sin discapacidad, pero siempre desde la valoración y el respeto de la diferencia. Este modelo aspira a potenciar el respeto por la dignidad humana, la igualdad y la libertad personal, propiciando la inclusión social, y asentándose sobre la base de determinados principios: autonomía 
personal, no discriminación, accesibilidad universal, normalización del entorno o dialogo civil, entre otros. De igual manera, apunta a la autonomía de la persona con discapacidad para decidir respecto a su propio proyecto de vida, Campoy, (2004) y De Asís, (2007).

En las consultas, se responde a la información demandada y se hace referencia a la educación sexual, cuya carencia es considerada esencial y fundamento de muchas de las cuestiones que nos plantean diariamente. Es importante remarcar que constantemente ha sido un tema polémico y complejo de emprender; sin embargo, hoy en día, este tipo de educación se reconoce como fundamental y de interés prioritario de las naciones, Organización de las Naciones Unidas para la Educación la Ciencia y la Cultura en 2010; Organización Panamericana de la Salud (OPS), Organización Mundial de la Salud (OMS) y Asociación Mundial de Sexología (WAS), en el 2000; entre otros y desde la Asesoría se reivindica poder llevar a cabo una pedagogía de la sexualidad según los cánones establecidos y consensuados por estos organismos.

El Modelo Biográfico profesional, Fallas, Artavia y Gamboa (2012), el otro marco en el que se basa la Asesoría, nace en Suiza a medidos de los años 50 del siglo pasado y es el que subyace en los planteamientos recogidos en las diversas normativas nacionales e internacionales sobre la educación sexual. Su carácter democrático viene dado por el profundo respeto que, desde la práctica, se tiene a la biografía de las personas, al defender la sexualidad como una construcción personal que debe estar basada en el conocimiento científico, la formación no sesgada y la libre elección Fallas, M (2009) La educación sexual, entendida desde este modelo educativo, se fundamenta en el bagaje científico y profesional, en una actitud positiva ante la sexualidad y la educación sexual, así como en el cultivo de una ética relacional, López, (2005). Pretende que las personas (en cualquiera de sus etapas evolutivas) vivan de manera responsable y saludable las diversas posibilidades de su sexualidad. Su fin último es que las personas a las que se imparte la educación sexual, se asuman como seres sexuados de una forma positiva, vivan su sexualidad de manera saludable y establezcan relaciones interpersonales gratificantes y no discriminatorias, Consejera de Educación, Cultura y Deporte (2008). Además, poder asesorar en sexualidad nos remite a la Ley Orgánica 3/2010 y el Real Decreto Legislativo 1/2013, de 29 de noviembre, por el que se aprueba el Texto Refundido de la Ley General de derechos de las personas con discapacidad y de su inclusión social, en el que se encomienda entre otras cosas, formar e informar y fomentar la investigación para generar conocimiento que beneficie a la sociedad.

\section{OBJETIVOS DE LA INVESTIGACIÓN}

\section{Objetivo General}

Registrar las solicitudes de asesoramiento para tener información de la naturaleza de las demandas y la implicación a la población que las realiza.

\section{Objetivos Específicos}

Describir en categorias las solicitudes recibidas en la Asesoria por parte de las personas con DF, sus familiares y los profesionales que les atienden

Presentar el tipo de relación establecida desde la Asociación para dar respuesta a las demandas solicitadas.

Dejar constancia de la importancia que ha ido tomando la asesoria en cuanto a aumento de demandas y temas a resolver en estos años.

Demostrar la importancia que para las personas con DF tiene la sexualidad por la necesidad de visibilizar sus demandas y resolver las necesidades en materia sexual de estas personas para empoderarlas. 


\section{SUJETOS DE ESTUDIO.}

Personas con diversidad funcional, familiares, cuidadores y profesionales de diversos ámbitos del territorio español que entraron en la web y consultaron sus dudas, problemas o situaciones personales en referencia a su sexualidad, etc., a traves de la Asesoria de ANSSYD. La mayoría de consultas se realizaron por via on line, telefónica y por skepy. La Asesoría cuando recibe una consulta remite la demanda al equipo de profesionales y estos se encargan de asignar a la persona que puede resolver el problema.

Los criterios de inclusión fueron en el caso de las PDF, no presentar una afectación cognitiva que les impidiera entender las explicaciones online, telefónicas o vía skype. En el caso de los familiares y profesionales, se han incluido todas las consultas, solo se pensó en excluir los casos que no fueran verdaderas consultas. No se dió ningún falso consultor.

\section{METODOLOGÍA}

Diseño Cuantitativo Descriptivo Transversal sobre las intervenciones llevadas a cabo por la Asesoría de ANSSYD a personas con DF, familiares y profesionales

Para llevar adecuadamente el registro de la información y cuantificación de los datos, se ha utilizado el paquete Microsofot Office 2010.

Solo se ha cuantificado el tema al que pertenece la pregunta en cuestión, no están registradas las sucesivas consultas necesitadas, en muchas ocasiones más de una, en función de la persona para resolver la duda planteada en el caso concreto. Se realiza un análisis descriptivo de las diferentes variables, haciendo registros separados en función de las dudas planteadas por profesionales y las consultadas por PDF, familiares y cuidadores.

Limitaciones del estudio: El anonimato de la asesoria ha sido una limitación para conseguir una mayoría de items así como las características propias de la investigación.

\section{ASPECTOS ÉTICOS}

Este proyecto se llevó a cabo teniendo en cuenta la legislación vigente y acatando las normas éticas de la Declaración de Helsinki y de Buena Práctica Clínica y de Laboratorio. El tratamiento, comunicación y la cesión de los datos de carácter personal de todos los sujetos participantes se ajusta a la Ley Orgánica española 15/1999 de 13 de diciembre de protección de datos de carácter personal, garantizando la confidencialidad a nivel de protección de la legislación vigente de nuestro país, Agencia Estatal Boletín Oficial del Estado. Ley Orgánica 15/1999, (2006).

\section{RESULTADOS}

Las consultas realizadas han ido en aumento año tras año. Se incluyen en el estudio las 3614 consultas realizadas a la Asesoria durante seis años en el periodo comprendido entre 2012 a 2017 , de las que 1856 son realizadas por familiares, cuidadores y personas con DF centrando sus dudas en las siguientes variables: Embarazo, desconocimiento sobre sexualidad, disfunciones sexuales, coito, orientación sexual, juguetes eróticos, asistencia sexual (AS), desconocimiento de las funciones de la AS equiparando esta figura a la de un profesional que imparte una terapia.(Tabla 1).

La mayoría de las consultas son online o telefónicas por lo que son estas las que se han registrado en el estudio. Las consultas por videoconferencia han sido consideradas como consultas online y se han integrado en ellas. La presencialidad ha sido casi inexistente por ser consultas realizadas desde diversos lugares del Estado. La dificultad de venir a la Asociación ha quedado de esta forma compensada, solo se ha recurrido a ella en contadas ocasiones, que no han sido consideradas relevantes a contabilizar e integrar en el presente trabajo.

Se ha considerado realizar una tabla única con los registros de consultas por parte de familia- 
res y de personas con diversidad funcional porque las preguntas formuladas son bastante homogéneas. En ella se detecta como aspecto relevante, la falta de información y de educación sexual.

Tabla 1: Asesoramiento a familiares y PDF

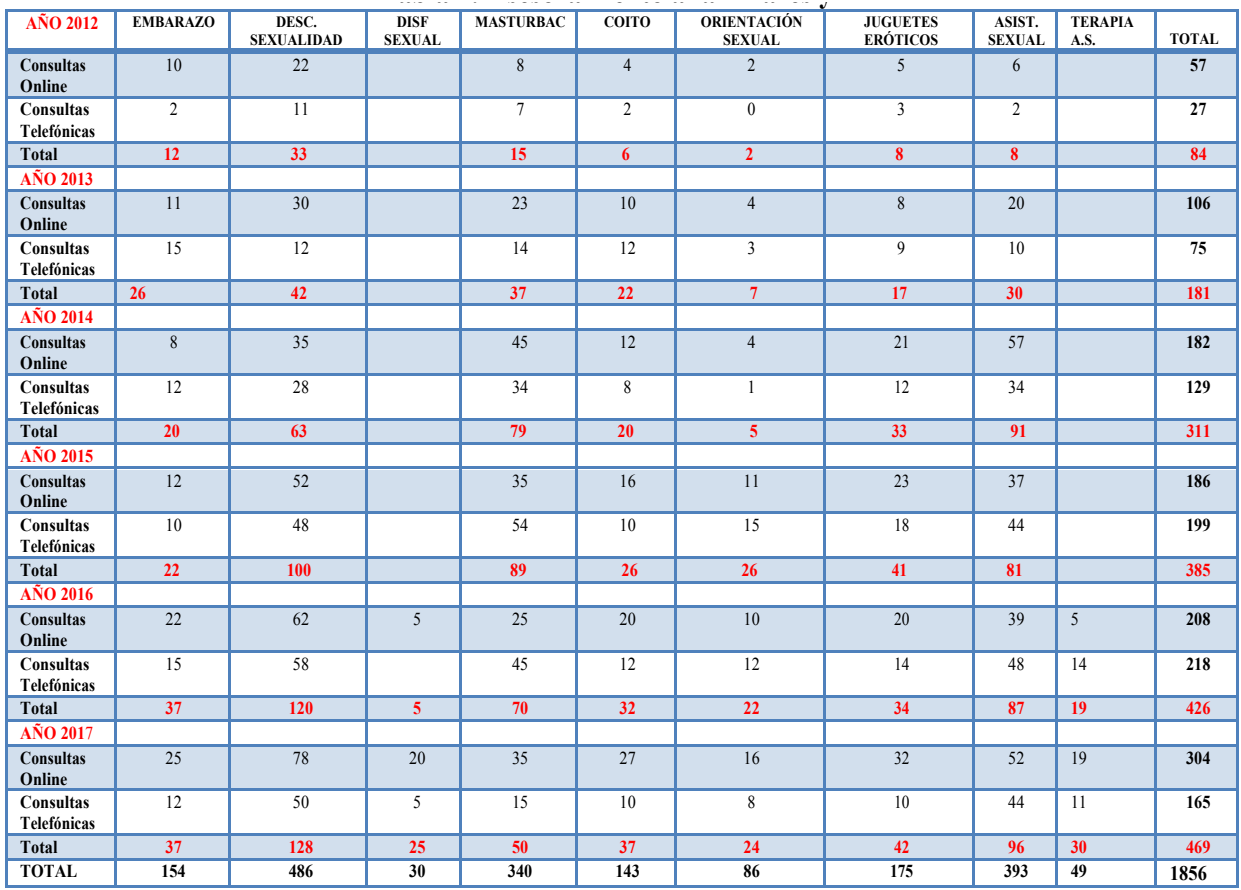

En la tabla2, se han descrito las consultas realizadas por los profesionales de diversos ámbitos que trabajan con personas con DF. Han sido un total de 1758, centrando sus dudas en las siguientes variables: anticoncepción, desconocimiento y dudas sobre sexualidad en PDF, masturbación, coito, orientación sexual, juguetes eróticos, asistencia sexual (AS), fantasías y derechos sexuales y reproductivos de las PDF.

En el caso de los profesionales no surge ninguna pregunta sobre el tema del embarazo, aspecto este que debe preocuparles menos, aspecto que se deduce por la ausencia de necesidad de información sobre el tema. En cambio en el caso de familiares y de personas con DF es un tema muy demandado y con mayor preocupación por las madres y los propios afectados.

La diferencia de casos de un año para otro es muy significativa en todas las categorías que han surgido a lo largo del periodo del estudio. Las preguntas sobre Asistencia Sexual son las de más reciente aparición, es en los dos últimos años en los que se plantean esta posibilidad, hasta entonces inexistente. 
Tabla 2: Consultas realizadas por profesionales

\begin{tabular}{|c|c|c|c|c|c|c|c|c|c|c|}
\hline AÑ̃ 2012 & ANTICONCEP & $\begin{array}{c}\text { DUDAS } \\
\text { SEXUALIDAD }\end{array}$ & MASTURBACIÓN & COITO & $\begin{array}{l}\text { ORIENTACIÓN } \\
\text { SEXUAL }\end{array}$ & $\begin{array}{l}\text { JUGUETES } \\
\text { ERÓTICOS }\end{array}$ & $\begin{array}{l}\text { ASISTENCIA } \\
\text { SEXUAL }\end{array}$ & FANTASIAS & DSR & TOTAL \\
\hline $\begin{array}{l}\text { Consultas } \\
\text { Telefónicas }\end{array}$ & 2 & 11 & 1 & 2 & 0 & 6 & 3 & & & 25 \\
\hline Total & 4 & 21 & 3 & 3 & 2 & 11 & 9 & & & 53 \\
\hline \multicolumn{11}{|l|}{ AÑO 2013} \\
\hline $\begin{array}{r}\text { Consultas } \\
\text { Telefónicas }\end{array}$ & 15 & 12 & 8 & 6 & 3 & 16 & 8 & & & 68 \\
\hline Total & 25 & 31 & 12 & 9 & 7 & 28 & 18 & & & 130 \\
\hline \multicolumn{11}{|l|}{ AÑO 2014} \\
\hline $\begin{array}{c}\text { Consultas } \\
\text { On line }\end{array}$ & 8 & 45 & 43 & 8 & 3 & 24 & 42 & & & 173 \\
\hline $\begin{array}{c}\text { Consultas } \\
\text { On line }\end{array}$ & 18 & 54 & 53 & 18 & 7 & 26 & 53 & & & 229 \\
\hline $\begin{array}{r}\text { Consultas } \\
\text { Telefónicas }\end{array}$ & 13 & 43 & 35 & 4 & 2 & 12 & 28 & 5 & & 142 \\
\hline Total & 31 & 97 & 88 & 22 & 9 & 38 & 81 & 5 & & 371 \\
\hline \multicolumn{11}{|l|}{ AÑO 2016} \\
\hline $\begin{array}{c}\text { Consultas } \\
\text { On line }\end{array}$ & 20 & 55 & 50 & 19 & 5 & 19 & 54 & 3 & 4 & 229 \\
\hline $\begin{array}{l}\text { Consultas } \\
\text { Telefónicas }\end{array}$ & 24 & 40 & 38 & 22 & 11 & 24 & 33 & 1 & 4 & 197 \\
\hline Total & 44 & 95 & 88 & 41 & 16 & 43 & 87 & 4 & 8 & 426 \\
\hline \multicolumn{11}{|l|}{ AÑO 2017} \\
\hline $\begin{array}{c}\text { Consultas } \\
\text { On line }\end{array}$ & 30 & 72 & 50 & 27 & 15 & 34 & 74 & 5 & 22 & 269 \\
\hline
\end{tabular}

\section{DISCUSIÓN}

A lo largo de la historia, las personas con diversidad funcional se han visto privadas de satisfacer sus necesidades sexuales. En nuestro país la mayoria de guías o programas de educación sexual dirigidos a personas con diversidad funcional están centrados en personas con discapacidad intelectual y muchas veces no cubren sus necesidades.

El presente trabajo de investigación, a tenor de las búsquedas bibliográficas realizadas para poder fundamentar los resultados presentados, no se han encontrado estudios previos que desarrollen la situación actual de la sexualidad de las PDF, solo algunas guías como la elaborada por López (2011) ,FEAPS Canarias (2015) y el Gobierno del Principado de Asturias (2016) que recomiendan realizar un estudio previo con el fin de adecuar la información a la realidad de partida, atendiendo aspectos como las necesidades expresadas, las características y opiniones personales ,el entorno familiar, la formación de los profesionales sobre este tema y los recursos disponibles.

Haciendo alusión a las carencias en educación sexual detectadas en el estudio, hemos de considerar tal como refiere Isler (2009)que uno de los pilares más importantes es el ámbito familiar y la mayoria de los padres con PDF no reconocen la sexualidad de sus hijos con diversidad funcional. Los resultados ponen de manifiesto que uno de los principales miedos que surge en los padres, cuando sus hijos muestran interés sexual es el miedo al embarazo no deseado,datos que coinciden con las aportaciones de Castelao,Schiavo, Jurberg (2003)y Varas (2011). En cuanto a las consultas realizadas por los profesionales implicados en la atención de las PDF los datos de nuestro estudio 
coinciden con las aportaciones realizadas por Castelo (2003) y Torices, García (2007) donde estos autores refieren la necesidad de sensibilizar a los profesionales para que ofrezcan información y orientación sobre sexualidad a las PDF. y a sus familiares

\section{CONCLUSIONES}

Los resultados de la investigación refieren que el número de consultas crece exponencialmente siendo la consulta más frecuente tanto en el grupo de familiares, cuidadores y personas don DF como en el de profesionales, la dudas sobre sexualidad, lo que indica la falta de información que sobre sexualidad tienen los grupos que consultan.

Las consultas que se realizan por via on line son más numerosas que por via telefónica en todas las categorias que han consultado ambos grupos, lo que implica que se prefiere utilizar esta forma nueva de comunicación para hacer preguntas que la directa oral por teléfono. Es posible el anonimato genere menos ansiedad y tranquilidad a la hora de exponerla.

Tanto en un grupo como en el otro se hace el mismo número de preguntas sobre la Asistencia Sexual, tema que va aumentando en los últimos 3 años, en los que este servicio sexual se ha publicitado sensiblemente con la publicación de artículos, programas radiofónicos y de TV, debates, charlas, conferencias que han suscitado una curiosidad y deseo de información o de utilización del servicio.

Es relevante tambien la importancia que ambos grupos dan a la reproducción, con las consultas sobre la planificación de la paternidad/maternidad y las posibilidades de esterilización y hasta que punto se respetan los deseos de las personas con DF.

En cambio la necesidad de recibir información sobre los derechos sexuales y reproductivos en ambos grupos es muy pequeña, solo los profesionales se interesan acerca de esta información todo y que en los últimos años se ha trabajado mucho para que estos derechos sean conocidos por todos, la realidad del trabajo presentado, nos indica que no solo no suscitan interés sino que no son conocidos por parte de los sujetos del estudio.

Las relaciones genitales con coito no son tampoco el tema más demandado, siendo un aspecto que preocupa más al grupo de familiares y personas con DF que a los profesionales.

La educación sexual es fundamental para el empoderamiento de las PDF se detecta en la mayoria de las consultas una carencia de Educación afectivo sexual y los datos reflejan la importancia de concienciar y educar a las PDF a familiares y profesionales en sexualidad

\section{BIBLIOGRAFÍA}

Boletín Oficial del Estado. Real Decreto 1393/2007, de 29 de Octubre, (2007) por el que se establece la ordenación de las enseñanzas universitarias oficiales. Boletín Oficial del Estado 260: 44037-44048.

Campoy, I. (2004) Los derechos de las personas con discapacidad. Perspectivas sociales, políticas, jurídicas y filosóficas. Madrid: Carlos III-Dykinson.

Castelao TB., Schiavo, MR., Jurberg P. (2004) Sexuality in Down syndrome individuals. Rev. saúde pública [Internet].; $37 \quad$ (1): 32-39. Disponible en: http://www.scielosp.org/ $\mathrm{pdf} / \mathrm{rsp} / \mathrm{v} 37 \mathrm{n} 1 / 13542 . \mathrm{pdf}$

Consejera de Educación, Cultura y Deporte. Dirección General de Ordenación e Innovación Educativa. (2008) Programa Harimaguada. Multimedia para la educación sexual, [Disco compacto]. Gobierno de Canarias.

Convención de las Naciones Unidas sobre los Derechos de las personas con discapacidad. (2006) Asamblea General de la Naciones Unidas.76ª sesión plenaria., New York. 
De Asís, R. y Palacios, A.(2007) Derechos humanos y situaciones de dependencia. Madrid: Universidad Carlos III-Dykinson.

Fallas, M., Artavia, C. y Gamboa, A.(2012) Educación sexual: Orientadores orientadoras desde el modelo biográfico y profesional. Revista Electrónica Educare. 53-71. Disponible en: http://www.revistas.una.ac.cr/index.php/EDUCARE/issue/view/418. Consultado 20 de Marzo de 2108

Fallas, M. (2009) Educación afectiva y sexual. Programa de formación docente de secundaria. (Tesis doctoral). Salamanca: Universidad de Salamanca.

García Ruiz M. (2009) Educación sexual y discapacidad. Talleres de educación sexual con personas con discapacidad. Material didáctico. [Internet]. Oviedo: Gobierno del Principado de Asturias; [citado el 1 de abril de 2017]. Disponible en: http://www.asturias.es/Asturias/descargas /PDF\%20DE\%20TEMAS/Asuntos\%20So ciales/guia\% 20de\%20educacion \%20sexual\%20y \%20discapacidad.pdf

Guatemala, Promoción de la salud sexual: Recomendación para la acción. Organización Panamericana de la Salud (OPS), Organización Mundial de la Salud (OMS) y Asociación Mundial de Sexología. (2000). Recuperada de http://www2.hu-berlin.de/sexology/ gesund/ archiv/ spanisch/ salud.htm.

Isler A, Beytut D, Tas F, Conk Z. (2009) A study on sexuality with the parents of adolescents with intellectual disability. Sex.disab.; (2): 229-237

Lameiras, M., Carrera, M. V. y Rodríguez, Y. (2008) Nuevos retos para la educación sexual: hacia un modelo integral. Sexología Integral.; 5(1): 35-38

López, F. (2005) La educación sexual. Madrid: Biblioteca Nueva.

López Sánchez F. (2011) Guía para el desarrollo de la afectividad y de la sexualidad de las personas con discapacidad intelectual. Desarrollo de la planificación y tutorización individual. La educación sexual en el ámbito de la discapacidad intelectual 36 [Internet]. Valladolid: Junta de Castilla y León, Consejería de Familia e Igualdad de Oportunidades, Gerencia de Servicios Sociales [citado el 5 de marzo de2018]. Disponible e $n$ : file:///D:/Documentos /ENFERMERÍA/TFG/Estudios/España/Programas\%20tabla/ Guía\%20afecti vidad\%20felix\%20lopez.pdf

Oliver, M. (1998) ¿Una sociología de la discapacidad o una sociología discapacitada?, En Barton Len, (Comp.). Discapacidad y sociedad. Madrid: Morata.:34-58.

Organización de las Naciones Unidas para la Educación la Ciencia y la Cultura. 2010.

Palacios A. (2008) El Modelo Social de la Discapacidad: Orígenes, características y plasmación en la Convención. Interacciones sobre los Derechos de las Personas con Discapacidad. CERMI. Madrid

Parra N, Oliva M.(2015) Sexualidades diversas. Manual para la atención de la diversidad sexual en las personas con discapacidad intelectual o del desarrollo. [Internet]. Canarias: Confederación Española de Organizaciones en favor de las Personas con Discapacidad Intelectual o del Desarrollo de Canarias (FEAPS Canarias); [citado el 15 de marzo de 2018]. Disponible en: file:///D:/Descargas/Sexualidades_Diversas_WEB_OK\%20(1).pdf

Real Decreto Legislativo (2013).1/2013, de 29 de noviembre. Texto Refundido de la Ley General de derechos de las personas con discapacidad y de su inclusión social. BOE.2013: 289

República China, Declaración de los derechos sexuales, Asociación Mundial para la Salud Sexual (WAS). (1999) Versión revisada y aprobada por la Asamblea General de la Asociación Mundial de Sexología celebrada en Hong Kong. Disponible en: http://www.ctv.es/ USERS/sexpol/derechos_sexuales.htm 
UNESCO. (2010) Orientaciones técnicas internacionales sobre educación sexual. Un enfoque basado en evidencia orientado a escuelas, docentes y educadores de la salud. Santiago de Chile. [citado el 15 de marzo de 2018]. Disponible en http://unesdoc.unesco.org/images/0018/001832/183281s.pdf

Varas Cortés J. (2011) Adolescentes con discapacidad psíquica: derechos en salud sexual y reproductiva. rev. obstet. ginecol.; 6 (3): 197-201

Victoria, J. A. (2013) El modelo social de la discapacidad: hacia una nueva perspectiva basada en los derechos humanos. Revista In Jure Anáhuac Mayab. 1(2): 143-158. 